\title{
NOUVELle
}

\section{Syndrome de Bardet-Biedl Une famille unique pour un gène majeur (BBS10)}

Hélène Dollfus, Jean Muller, Corinne Stoetzel, Virginie Laurier, Dominique Bonneau, André Mégarbané, Olivier Poch, Jean-Louis Mandel

> Décrit en 1920, le syndrome de Bardet-Biedl (BBS) (OMIM 209900) est une maladie autosomique récessive caractérisée par un cortège clinique comportant comme signes cliniques principaux : rétinopathie pigmentaire précoce, obésité, polydactylie, hypogonadisme, déficit cognitif variable et anomalies rénales $(\rightarrow)$. Cette affection, a priori clairement

$(\rightarrow) \mathrm{m} / \mathrm{s} 2006$, n० 6-7, p. 576

définie sur le plan clinique, a été un des premiers exemples d'hétérogénéité génétique prouvée par cartographie génétique par homozygotie au début des années 1990. Récemment, il a été suggéré que l'hérédité du syndrome pouvait se démarquer de l'hérédité autosomique classique avec, pour certaines familles, la description de trois allèles mutés dans deux gènes BBS différents (hérédité oligogénique ou triallélique)

[1] $(\rightarrow)$, le troisième allèle induisant un effet épista- $(\rightarrow) \mathrm{m} / \mathrm{s} 2002$, $n^{\circ} 12$, p. 1205 cellulaire planaire pour certains épithéliums, mais également dans d'autres tissus comme le tissu nerveux. Le syndrome BBS est maintenant classé comme une ciliopathie au même titre que les polykystoses rénales et les néphronophtises, le syndrome d'Alström, le syndrome orofaciodigital de type 1 et plus récemment les syndromes de Meckel et de Joubert (pour revue, voir $[5,6])$.

Fin 2005, 9 gènes avaient été identifiés (BBS de 1 à 9), ne rendant compte que de $50 \%$ des patients. Le gène $B B S I$ est muté chez $20 \%$ à $25 \%$ des patients. Les gènes BBS de 2 à 9 ne sont retrouvés chacun mutés que dans un petit pourcentage de patients. Récemment, le gène BBS10 a été identifié par notre groupe et son impact majeur dans la maladie a été démontré [7]. Le gène $B B S 11$, impliqué dans une seule famille (mutation faux-sens) [8], ne nous paraît pas parfaitement validé, d'autant plus qu'il a été également retrouvé muté dans une forme de myopathie progressive (LGM2H).

gene modificateur interagissant avec les tions des autres gènes $B B S$ vient ainsi d'être décrit [2]. La physiopathogénie de la maladie a été révélée par la publication en 2003 du gène $B B S 8$, identifié comme codant une protéine du centrosome ou du corpuscule basal impliquée dans la constitution du cil primitif [3]. Les cils primitifs, présents dans une grande majorité de nos cellules, sont impliqués dans l'exploration de l'environnement interne et externe de l'organisme par le biais de fonctions chimio-, photo- et mécanosensorielles [4]. De plus, les cils jouent un rôle essentiel dans des étapes critiques du développement embryonnaire, et notamment dans le phénomène de polarité

\section{Un dixième gène pour BBS}

L'histoire du gène BBSIO commence par l'exploration clinique d'une famille hautement consanguine avec 5 fratries atteintes de BBS, issue d'un petit village du Nord du Liban, et dont l'étude s'est faite en collaboration avec l'Université St Joseph de Beyrouth (Figure 1A). L'approche classique de cartographie par microsatellites a échoué, entraînant le choix d'une stratégie de cartographie par homozygotie utilisant les puces SNP 10K Affymetrix (ces puces permettent
H. Dollfus, C. Stoetzel, V. Laurier :

Laboratoire de génétique médicale EA3949, Faculté de Médecine, Université Louis Pasteur, Hôpitaux Universitaires de Strasbourg, Hôpital de Hautepierre, avenue Molière, 67098 Strasbourg Cedex, France.

J. Muller : Laboratoire de Biologie et génomique intégratives, IGBMC, 1, rue Laurent Fries, 67404 IIIkirch, France. Laboratoire de Biologie moléculaire, d'Analyse génique et de modélisation, Centre de Recherche Public-Santé,

L1911 Luxembourg, Luxembourg.

0 . Poch : Laboratoire de Biologie et génomique intégratives, IGBMC, 1, rue Laurent Fries, 67404 Illkirch, France.

D. Bonneau : Département de Génétique, CHU d'Angers, 4, rue Larrey, 49033 Angers Cedex 01, France.

A. Mégarbané : Laboratoire de Génétique, Université Saint-Joseph, Beyrouth, Liban.

J.L. Mandel : Université Louis Pasteur,

Faculté de Médecine, 67000 Strasbourg, France. IGBMC (Institut de Génétique et de Biologie Moléculaire et Cellulaire), Département de génétique humaine. Inserm U596, CNRS UMR 7104, Collège de France, Chaire de génétique humaine, 67400 Illkirch, France. Helene.Dollfus@chru-strasbourg.fr

l'analyse de 10000 SNP répartis sur l'ensemble du génome, représentant un gain d'informativité d'environ 8 fois par rapport aux 400 marqueurs microsatellites utilisés en général pour réaliser «un tour de génome»). Toutefois, au lieu d'identifier un seul locus, comme attendu pour une telle famille, nous avons trouvé deux zones d'intérêt: une première zone sur le chromosome 16 correspondant à la région du gène BBS2 (pour une fratrie) et une autre zone sur le chromosome 12 (pour 3 fratries), ne correspondant à aucun gène $B B S$ connu (Figure IB).

Une mutation faux-sens a été identifiée dans BBS2 pour deux sœurs homozygotes dans la région correspondante en $16 q 21$, mais cette mutation n'était pas présente chez les autres patients de cette famille. Ce premier résultat a confirmé une faille dans l'approche classique de cartographie par homozygotie [9]. 
Dans la région du chromosome 12, 8 gènes ont été retenus comme prioritaires soit par leur présence dans une liste de gènes conservés dans les organismes ciliés ou en raison de leur fonction. Dans un de ces gènes, FLJ23560 (dont l'annotation dans les bases de données du génome était très minime et partiellement erronée), une mutation faux sens (S311A) a été identifiée

\section{III.8}

A
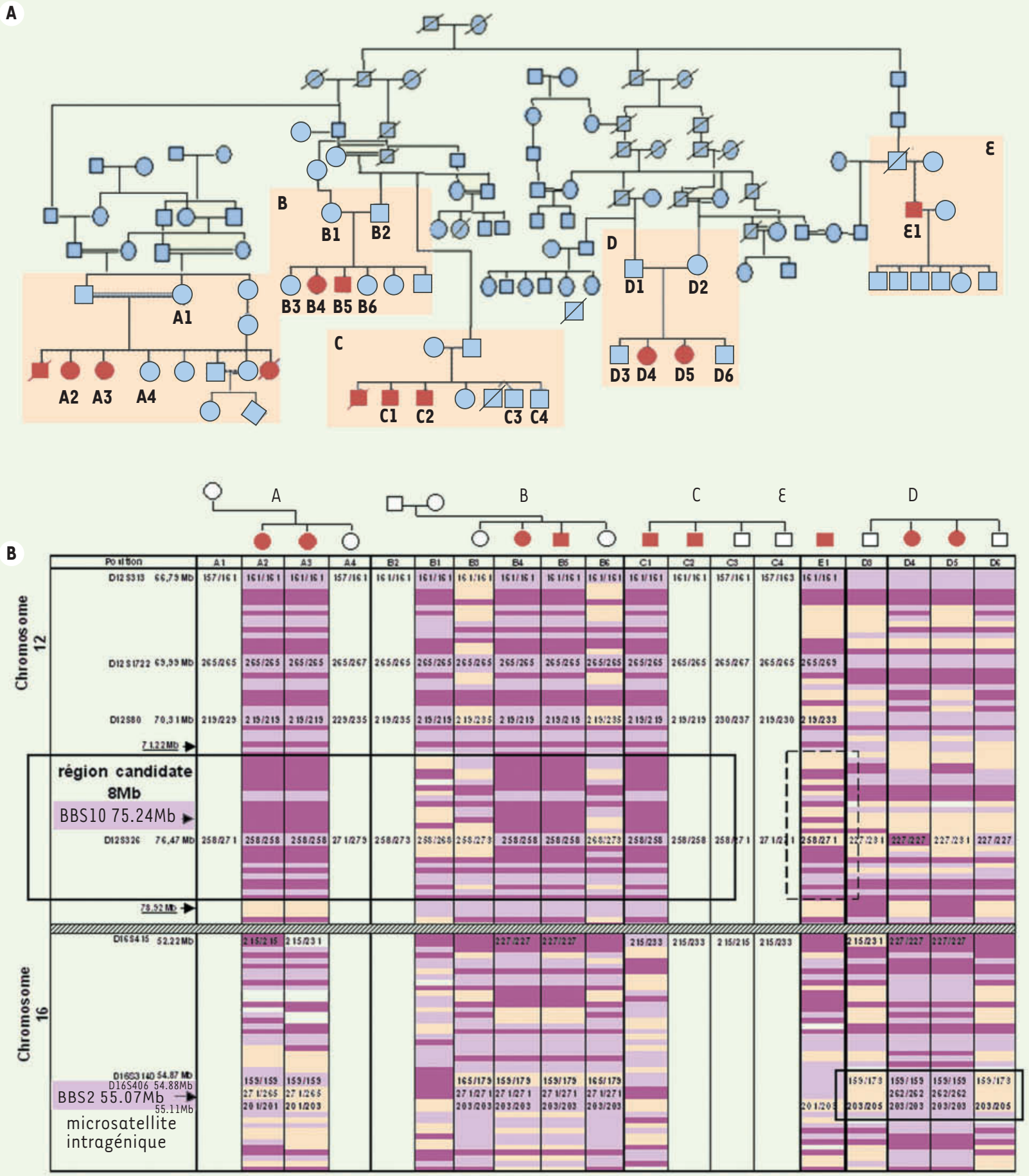

Figure 1. A. Arbre généalogique de la famille libanaise. B. Cartographie par homozygotie fondée sur les SNP avec, de plus, les marqueurs microsatellites de la région qui ont contribué à définir deux zones d'intérêt. En mauve et en rouge sont représentés les SNP homozygotes (respectivement $A A$ et $B B$ ), les régions en jaune indiquent les allèles hétérozygotes $(A B)$. 


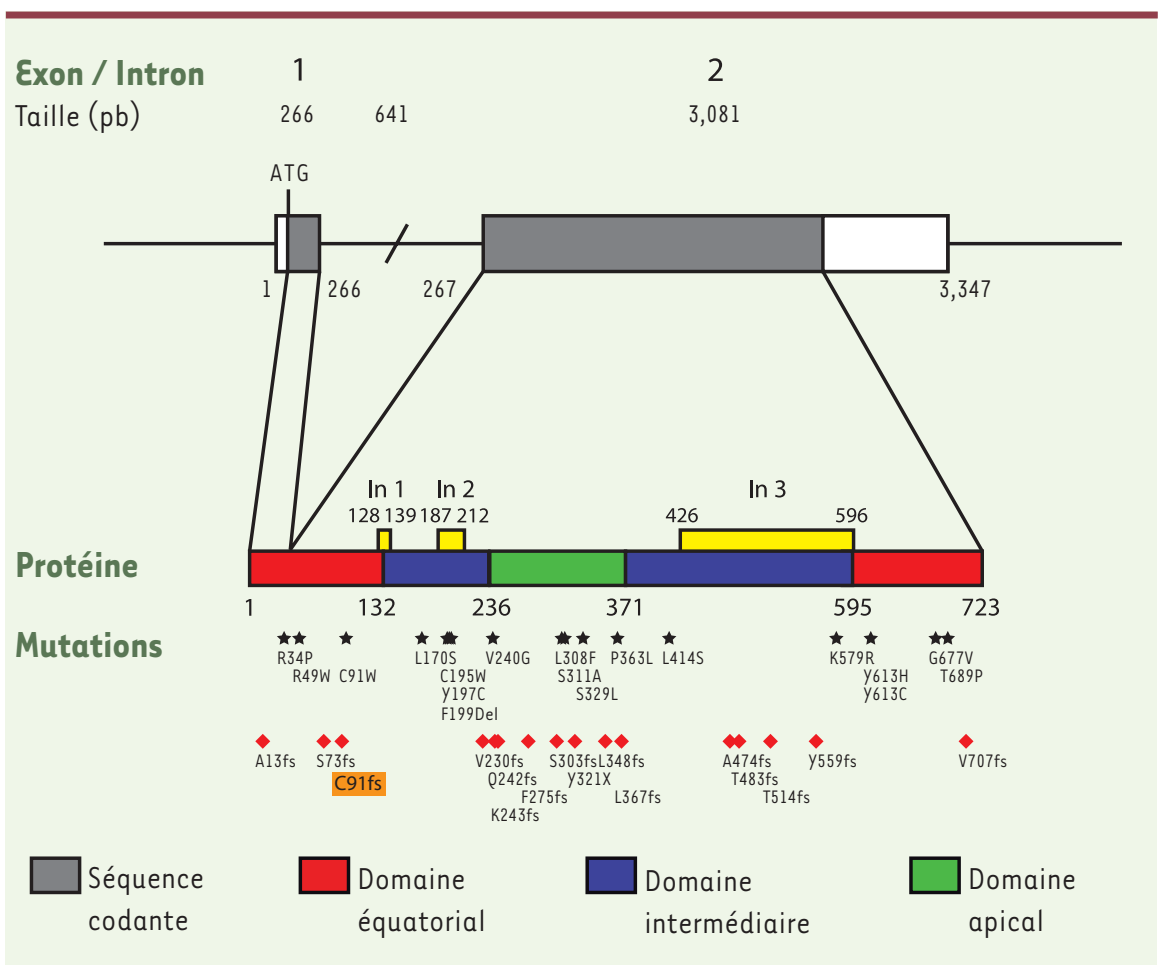

Figure 2. Le gène BBS10 avec la position et la nature des mutations. A. Séquence génomique de BBS10. $B$. Protéine avec les domaines connus indiqués par différentes couleurs. Les insertions dans le domaine intermédiaire sont représentées par les boîtes jaunes au-dessus de la protéine. Les mutations retrouvées dans les familles sont indiquées sous la protéine, les étoiles indiquant des faux-sens et les losanges des mutations tronquantes (frameshifts ou non-sens). La mutation récurrente est représentée en orange.

pour les 6 patients homozygotes correspondant aux 3 fratries liées à cette région, et de manière inattendue, à l'état hétérozygote, en combinaison avec une autre mutation, chez le patient unique d'une autre fratrie). L'analyse de ce gène dans les familles BBS de la cohorte française a révélé plusieurs mutations tronquantes, indiquant que FLJ23560 représentait effectivement un nouveau gène BBS (Figure 2) [7].

Pour explorer de manière exhaustive ce gène, nous avons élaboré une collaboration avec le Dr N. Katsanis (John Hopkins University, Baltimore, États-Unis) et avec le Dr P. Beales (Institute of Child Health, Londres, RoyaumeUni) : 311 familles de différentes origines ont été séquencées pour ce gène, y compris les familles ayant des mutations dans les gènes $B B S$ déjà connus. Nous avons trouvé des altérations pathogènes chez plus de $20 \%$ des familles. Ces résultats montrent que le gène $B B S 10$ est un gène majeur du syndrome, muté aussi fréquemment que le gène $B B S 1$. Des patients de 53 familles étaient porteurs de 2 mutations BBS10, alors
BBS10 : une chaperonine

pas comme les autres

Afin de mieux caractériser le gène BBS10 au niveau fonctionnel et évolutif, une étude bio-informatique exhaustive englobant les aspects phylogénétiques, génomiques et structuraux a permis d'établir des conclusions majeures sur la famille de ce gène.

Sur le plan fonctionnel, les recherches dans les banques de données de séquences ont révélé que le gène $B B S 10$, comme le gène $B B S 6$, appartient à la famille des chaperonines de type II. Ainsi nous avons pu établir qu'il existe au moins 2 gènes de cette famille qui sont impliqués dans la maladie. Les chaperonines de type II, présentes depuis les bactéries jusqu'à l'homme, sont des protéines multidomaines, organisées en complexe multiprotéique et impliquées dans le repliement et la conformation spatiale de protéines cibles. D'un point de vue structural, bien que l'architecture classique en 3 domaines (apical, intermédiaire et équatorial) des chaperonines soit conservée, BBS10 se distingue de toutes les chaperonines par la présence de 3 insertions de longueur variables (Figure 2). Sur la base de la structure 3D d'une chaperonine d'archaebactérie, les analyses structurales réalisées ont montré que ces insertions se situent en proximité spatiale émergeant sur la même face de la molécule. Ces éléments suggèrent l'existence d'un nouveau domaine fonctionnel discontinu ainsi que la spécialisation de BBS10 au regard des autres chaperonines. En raison de la contrainte structurale induite, il semble que BBS10, tout comme cela a été démontré pour BBS6, ne puisse former le complexe multiprotéique canonique à 8 sous unités des chaperonines. L'ensemble de ces observations indique que cette protéine définit une nouvelle sous-famille de chaperonines.

Sur le plan évolutif, BBS10 se distingue fondamentalement de l'ensemble des autres gènes BBS connus. En effet, l'analyse de la distribution du gène BBS10 au sein des organismes dont la séquence du génome est connue a permis de restreindre son existence aux seuls organismes vertébrés, suggérant une apparition récente de ce gène. De plus, de tous les gènes connus responsables de la maladie, c'est également le plus variable reflétant certainement une évolution rapide (ou une contrainte évolutive plus 
faible). Tous ces éléments amènent à penser que le gène BBS10 constitue une fonction récemment acquise par la cellule au sein de laquelle il pourrait jouer un rôle hautement spécialisé dans la mise en place des cils en s'adaptant aux différents contextes tissulaires présents chez les vertébrés.

Pour analyser la fonction du gène BBS10, des études ont été effectuées sur le poisson zèbre en inhibant l'expression à l'aide d'oligonucléotides de type morpholino (MO) spécifiques de BBS1O (équipe de N. Katsanis, John Hopkins University). L'injection d'une série de concentrations différentes de MO dans les embryons a montré des défauts dosage-dépendants des processus précoces des mouvements de la gastrulation, similaires aux phénotypes d'inhibition des autres gènes $B B S$. Ces résultats sont en accord avec l'hypothèse récemment développée de l'implication des gènes $B B S$ dans la polarité planaire cellulaire [10].
L'identification de BBS10 comme un gène impliqué dans plus de $20 \%$ des cas a un impact sur le diagnostic moléculaire de l'affection et le conseil génétique. La caractérisation du rôle de BBS10 au niveau du cil primitif permettra de disséquer la physiopathologie du complexe cilaire dans cette maladie qui induit un polyhandicap. $\diamond$ Bardet-Biedl syndrome : a unique family for a major gene (BBS10)

\section{REMERCIEMENTS}

Ministère de la recherche (PHRC 2002), RETINA

France, Lions Club du Kochersberg, PNRV INSERM

2005, Collège de France. Services de séquençage

et plateforme Affymetrix de l'IGBMC.

\section{RÉFÉRENCES}

1. Katsanis N, Ansley SJ, Badano JL, et al. Triallelic inheritance in Bardet-Biedl syndrome, a Mendelian recessive disorder. Science $2001 ; 293: 2256-9$.

2. Badano JL, Leitch CC, Ansley SJ, et al. Dissection of epistasis in oligogenic Bardet-Biedl syndrome. Nature 2006 ; 439 : 326-30.
3. Ansley SJ, Badano JL, Blacque $0 \varepsilon$, et al. Basal body dysfunction is a likely cause of pleiotropic BardetBiedl syndrome. Nature 2003 ; 425 : 628-33.

4. Pazour GJ, Witman GB. The vertebrate primary cilium is a sensory organelle. Curr Opin Cell Biol 2003; $15: 105-10$.

5. Badano JL, Mitsuma N, Beales PL, Katsanis N. The ciliopathies: an emerging class of human genetic disorders. Annu Rev Genomics Hum Genet 2006 ; $7: 125-48$.

6. Sayer JA, Otto EA, O'Toole JF, et al. The centrosomal protein nephrocystin- 6 is mutated in Joubert syndrome and activates transcription factor ATF4. Nat Genet 2006 ; $38: 674-81$.

7. Stoetzel C, Laurier V, Davis $\varepsilon \varepsilon$, et al. BBS10 encodes a vertebrate-specific chaperonin-like protein and is a major BBS locus. Nat Genet 2006 ; 38 : 521-4.

8. Chiang AP, Beck JS, Yen HJ, et al. Homozygosity mapping with SNP arrays identifies TRIM32, an E3 ubiquitin ligase, as a Bardet-Biedl syndrome gene (BBS11). Proc Natl Acad Sci USA 2006 ; 103 : 6287-92.

9. Laurier V, Stoetzel C, Muller J, et al. Pitfalls of homozygosity mapping: an extended consanguineous Bardet Biedl syndrome family with two mutant genes (BBS2, BBS10), three mutations, but no triallelism. Eur $J$ Hum Genet 2006 (sous presse).

10. Ross AJ, May-Simera H, Eichers ER, et al. Disruption of Bardet-Biedl syndrome ciliary proteins perturbs planar cell polarity in vertebrates. Nat Genet 2005 ; 37 : $1135-40$

\section{NOUVELLE}

Voie Wnt/B-caténine
et zonation métabolique du foie
Un nouvel acteur pour un ancien concept
Samira Benhamouche, Thomas Decaens,
Christine Perret, Sabine Colnot

\author{
Institut Cochin, CNRS UMR 8104, Inserm U567, \\ Université Paris V-René Descartes, \\ Faculté de Médecine, Institut Cochin, \\ 24, rue du Faubourg-Saint-Jacques, \\ 75014 Paris, France. \\ scolnot@cochin.inserm.fr
}

> Le rôle fonctionnel du foie est essentiellement métabolique, et consiste à trier et traiter les nutriments bruts provenant de l'intestin par la veine porte, pour biotransformer, synthétiser et sécréter nutriments, énergie, xénobiotiques et hormones qui seront délivrés aux différents organes par la veine hépatique, et à l'intestin par les canaux biliaires. L'unité fonctionnelle hépatique décrite par Kiernan dès 1833, qu'il s'agisse selon les auteurs du lobule, de l'acinus ou de la travée hépatocytaire (Figure $1 A$ et $1 B$ ), est orientée par le flux sanguin traversant le foie. La première rangée d'hépatocytes dits périportaux est alimen- tée en sang mixte provenant de l'artère hépatique et de la veine porte; elle est en contact avec les canaux biliaires. À l'opposé de la travée hépatocytaire, les hépatocytes dits périveineux entourent une veine centrolobulaire dans laquelle se déverse le sang efférent.

Il y a une trentaine d'années, K. Jungermann montrait, par des immunolocalisations des enzymes clés du métabolisme glucidique, que les hépatocytes étaient spécialisés différemment selon leur localisation le long de l'axe porto-central de la travée hépatocytaire. II introduisait ainsi le concept dynamique de zonation métabolique [1], qui permettait d'expliquer comment des fonctions, a priori opposées comme la biosynthèse et la dégradation d'un même nutriment (Figure 1B), pouvaient cohabiter dans le foie.

Au cours des dernières décennies cependant, l'identification de la mécanistique pouvant être mise en jeu pour assurer cette compartimentation des fonctions hépatiques fut souvent étudiée mais jamais résolue. C'est en étudiant des souris modélisant une forme fréquente d'hépatocarcinome humain consécutif à une activation aberrante de la voie Wnt/ $\beta$-caténine que nous avons pu constater que plusieurs protéines 